\title{
Diacronie
}

Studi di Storia Contemporanea

$\mathrm{N}^{\circ} 24,4 \mid 2015$

Le dittature militari: fisionomia ed eredità politica

\section{Physionomies et héritages de la dictature pinochétiste : du terrorisme d'État à la mise sous tutelle du processus transitionnel (1973-2015)}

\section{Nicolas Prognon}

\section{OpenEdition}

\section{Journals}

Édition électronique

URL : http://journals.openedition.org/diacronie/3790

DOI : 10.4000/diacronie.3790

ISSN : 2038-0925

Éditeur

Association culturelle Diacronie

Référence électronique

Nicolas Prognon, « Physionomies et héritages de la dictature pinochétiste : du terrorisme d'État à la mise sous tutelle du processus transitionnel (1973-2015) », Diacronie [En ligne], № 24, 4 | 2015,

document 13, mis en ligne le 29 décembre 2015, consulté le 06 mai 2019. URL : http://

journals.openedition.org/diacronie/3790; DOI : 10.4000/diacronie.3790 


\section{Diacronie}

\section{Physionomies et héritages de la dictature}

pinochétiste: du terrorisme d'Etat à la mise sous tutelle du processus transitionnel (1973-

\section{5)}

\section{Nicolas PROGNON *}

Le 11 septembre 1973, une junte militaire placée sous l'autorité du général Augusto Pinochet renverse le gouvernement de Salvador Allende. Ce régime militaire, en place jusqu'en mars 1990 lors de l'intronisation du président Patricio Aylwin, a réussi à consolider son pouvoir en supprimant les institutions existantes et en promulguant une nouvelle constitution en 1980. Puis le " président » Pinochet orchestre la sortie du régime et il décide de contrôler la gestion de la transition vers la démocratie en verrouillant le système politique. Pour autant, alors que ce pays commémorait les quarante ans du coup d'Etat en 2013, les stigmates de cette transition sous tutelle de 1990 et l'héritage politique de la dictature restent clivant pour la société chilienne.

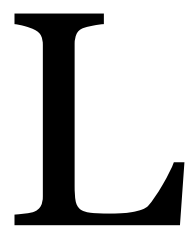

e 11 septembre 1973, une junte militaire placée sous l'autorité du général Augusto Pinochet renverse le gouvernement de Salvador Allende, le Chili ouvre alors une parenthèse autoritaire aux incidences encore perceptibles de nos jours. Jusqu'à cette date, la stabilité politique de ce pays est quasi légendaire dans le contexte latino-américain avec une armée ayant la réputation d'être la garante de la démocratie en regard de son caractère professionnel et constitutionnel; néanmoins si l'on se penche sur l'histoire nationale de ce pays, force est de constater que cet apolitisme relève du mythe ${ }^{1}$. Avec le coup d'Etat se met en place une dictature

${ }^{1}$ ROUQUIÉ, Alain, L’Etat militaire en Amérique latine, Paris, Le Seuil, 1982, p. 479. 
militaire qualifiée de pinochétiste puisque l'histoire de ce régime est intimement liée au personnage d'Augusto Pinochet et à son empreinte sur son évolution. Pour autant, il ne s'agit pas ici de retracer la trajectoire personnelle de ce dictateur ou de nous interroger sur sa personnalité mais de montrer son influence dans la structure même de la dictature, qui sévit au Chili de 1973 à 1989, puis au cours des deux décennies suivant l'avènement de la transition vers la démocratie. Son emprise peut s'entendre selon la formule de Stéphane Boisard: «le général Pinochet peut affirmer, après quelques années d'exercice du pouvoir seulement que «pas une feuille ne bouge au Chili sans qu'il le sache»².

La junte de gouvernement opère en plusieurs étapes un processus d'institutionnalisation. Dans un premier temps, démunie de programme économique, elle applique son projet politique inspiré par la doctrine de la sécurité nationale et cette phase s'accompagne d'une personnalisation du pouvoir autour du général Pinochet. La seconde étape complète la précédente et elle est marquée par la préparation, l'adoption par référendum et la mise en pratique graduelle de la nouvelle constitution. L'ultime période est celle de la transition vers la démocratie dont le déroulement est dicté par la loi fondamentale. Nous nous intéresserons plus précisément, dans ce cadre chronologique, au phénomène de politisation du régime militaire, à la consolidation du pouvoir et à sa gestion de la transition institutionnelle à la démocratie qui va demeurer jusqu'en 2006 sous tutelle des forces armées et au-delà circonscrite par les «enclaves autoritaires»3.

\section{Du terrorisme d'Etat à la nature personnaliste du régime (1973-1979)}

\subsection{La doctrine de sécurité nationale, projet politique du terrorisme d'Etat}

Le coup d'Etat frappe par sa violence aveugle et symbolique afin de montrer la rupture avec le régime antérieur, l'Unité populaire certes, mais également la démocratie parlementaire. Pour ce faire, les avions de la Fach bombardent le palais de la Moneda, incarnation du pouvoir politique civil. Cette répression furieuse trouve sa justification dans la lutte contre la subversion menée par des organisations de gauche,

2 BOISARD, Stéphane, HEREDIA, Marina, "Regards croisés sur les dictatures argentine et chilienne des années 1970", in Vingtième siècle, 105, 1/2010, pp. 109-126, p. 125.

3 GARRETON, Manuel Antonio, «La transformation de la société latino-américaine et les processus de démocratisation», in Cahiers du GELA-IS, 1, 2001, pp. 65-98. 
ainsi la Junte conçoit un ennemi interne. Elle entame alors une croisade destinée à sauver le pays d'un «Plan Z», construit de toute pièce, qui est décliné dans le «livre blanc» expliquant les raisons de son intervention4. Immédiatement, les fondements politiques de la terreur peuvent être appliqués. Ces derniers trouvent leur fondement idéologique dans le contexte de Guerre froide. Initialement, les Etats Unis tissent un réseau continental pour se protéger du communisme en signant le Traité interaméricain d'assistance réciproque (TIAR) à Rio en 1947 et la Charte de Bogota en 1948. Puis à partir des années 1950, l'intensification du conflit Est-Ouest pousse les Etats Unis à renforcer la formation militaire et l'apport de matériel de guerre pour l'Amérique latine pour éviter tout rapprochement entre les pays du continent et le bloc soviétique. Entre 1950 et 1965, 2.064 militaires chiliens se sont entrainés aux Etats Unis et 549 dans la zone du Canal de Panama, parmi lesquels Augusto Pinochet et quelques futurs membres de la Dirección de Inteligencia Nacional, plus connue sous son acronyme DINA, Manuel Contreras, Eduardo Iturriaga Neumann et Miguel Krassnoff. Ces chiffres placent le Chili au second rang, dans ce domaine, après le Brésil (3.632) et avant le Pérou (2.306)5. Dès la décennie 1960, les officiers chiliens appréhendent les conflits armés sous l'angle de la doctrine de sécurité nationale en se plaçant au centre du conflit politique de la sociétée . Ainsi, l'armée chilienne s'appuie sur un arsenal idéologique consistant en une «guerre sainte» anticommuniste, nationaliste et patriotique contre un régime illégitime et subversif. Toutes les violations des droits de l'homme sont permises au nom de la raison d'Etat pour éliminer, selon les termes du général Pinochet, le «cancer marxiste» responsable de la guerre civile7.

Les mois suivant le coup d'Etat sont marqués par une répression massive et peu sélective tant la notion d'ennemi intérieur recoupe de possibilités d'inculpation. La violence de l'intervention a un double but: étouffer les courants militaires pro-UP et anéantir la résistance armée des opposants civils. Cependant, l'absence de résistance et de préparation au coup d'Etat démontrent le contraire ${ }^{8}$. Des centaines d'individus sont exécutés et il faut attendre la fin de l'année 1973 pour que la Junte dispose

\footnotetext{
4 Libro Blanco del cambio en Chile, Santiago de Chile, Secretaría General de Gobierno - Ed. Lord Cochrane, 1973.

5 VALDIVIA ORTIZ DE ZARATE, Veronica, El golpe después del golpe. Leigh vs. Pinochet. Chile 1960-1980, Santiago, LOM Ediciones, 2003, p.32.

${ }^{6}$ Chiffres cités par GARATE, Emmanuel, La "Révolution économique" au Chili. A la recherche de l'utopie néoconservatrice 1973-2003, Paris, Ecole des Hautes Etudes en Sciences Sociales (EHESS), 2010, p. 172.

7 PINOCHET, Augusto, Camino recorrido. Memorias de un soldado, t. II, Santiago de Chile, Instituto Geográfico Militar, 1991. p. 18.

8 OSORIO REYES, Victor, «Mitos y mentiras del golpe militar», in La Huella, 12, 2002, pp. 223.
} 
d'infrastructures pour accueillir un nombre croissant de prisonniers. Des camps de concentration et des prisons sont installés dans le pays et des opérations de nettoyage s'organisent.

La guerre contre-subversive met à contribution l'ensemble des services de sécurité et de renseignement pour l'élimination des derniers bastions de résistance qui rivalisent de zèle dans les tâches de répression. Le Service de renseignements de la force aérienne (SIFA) prend pour cible le MIR et transforme son centre d'opération, l'Académie de Guerre Aérienne, en centre de torture; ses résultats sont probants avec, en octobre 1974, l'exécution du secrétaire général du MIR, Miguel Enriquez. A la suite de ce succès, il est rebaptisé DIFA (Direction des Renseignements de la force aérienne) et oriente ses activités vers le renseignement et l'infiltration. Une de ses sections collabore alors avec le Service de renseignement naval (SIN), le Département de renseignement de l'armée (DINE) et la Division de renseignements des carabiniers (DICAR). Ce regroupement de circonstance devient le Commando Conjoint dissout en 1977; sa mission est d'infiltrer et de démanteler les partis clandestins. En ce sens, des milliers de personnes vont être fichées et surveillées. Une des particularités de cette phase de liquidation de l'opposition ou de «simple travail de nettoyage»9 est la concurrence entre les services de renseignement des différentes armes. Aussi, le Commando Conjoint dispute la palme de la répression à une autre émanation du pouvoir militaire, la DINA ${ }^{10}$. Elle est créée par le décret-loi $n^{\circ} 521$ du 14 juin 1974, elle est dirigée par Manuel Contreras et elle dépend entièrement du gouvernement militaire. Cette police politique dispose de pouvoirs démesurés en termes de violations des droits de l'homme. Elle met en place une structure complexe organisée autour de centres de torture à l'existence éphémère pour ne pas éveiller les soupçons, de centres d'interrogatoire et de cliniques pour soigner les personnes que l'on souhaite maintenir en vie. Pour arriver à leurs fins, les agents ont recours aux pratiques les plus sadiques pour briser moralement et/ou physiquement leurs victimes ${ }^{11}$. Rapidement, les opposants à la Junte sont décimés et les organisations clandestines doivent se prémunir contre les délations et les infiltrations des membres des services de renseignements. L'activité de la DINA ne se limite pas à la traque des ennemis de l'intérieur au Chili car elle participe à des actions extérieures dans le cadre de

\footnotetext{
9 Général Pinochet, extrait d'un discours prononcé le 11 septembre 1979, cité dans El Mercurio, 12 de septiembre 1979.

${ }^{10}$ Consulter: SALAZAR, Manuel, Las letras del horror - 1, la DINA, Santiago, Ediciones LOM, 2011.

${ }^{11}$ Lire le témoignage édifiant de ARCE, Luz, L'enfer, terreur et survie sous Pinochet, Paris, Les petits matins, 2013 .
} 
l'Opération Condor ${ }^{12}$. Ironie de cette histoire de la terreur, l'assassinat d'Orlando Letelier, ex-ministre d'Allende et personnage clef de l'opposition à Pinochet, dans le centre de Washington le 21 septembre 1976 par la DINA pour Condor, provoque sa dissolution et sa substitution, sur décision de la junte, par la Centrale Nationale d'Information (CNI) en août 1977. Désormais, la répression se mue en une guerre psychologique toujours conforme aux dogmes de la sécurité nationale.

\subsection{La suppression des institutions existantes et la personnalisation du régime}

De façon synchrone, la dictature dans sa refonte de la société chilienne, telle qu'elle est énoncée dans la déclaration de principes de mars 1974, institutionnalise le régime et progressivement, ce dernier va s'incarner dans la figure du commandant en chef de l'armée de terre. Dans son souci de restaurer la «chilénité» perdue, la junte se munit d'un appareil juridico-répressif pour asseoir son contrôle sur la population. «Le processus qui débute le 11 septembre 1973 est fortement marqué par une concentration des pouvoirs par la Junte de gouvernement et surtout par le président de la République. Ensemble, ils exercent les pouvoirs constituants, législatifs et exécutifs ${ }^{13}$.

Composée des quatre généraux en chef de l'armée ${ }^{14}$, elle montre un grand attachement à la légitimation du coup d'Etat. Les militaires estiment qu'Allende a violé les droits individuels fondamentaux, qu'il a interprété la constitution de 1925 dans un esprit totalitaire, qu'il a outrepassé ses droits en ne respectant pas les décisions du Congrès et de la Cour Suprême et qu'il a manqué d'autorité à l'égard des partis et des organisations populaires, ce qui était de nature à détruire le caractère présidentiel du régime ${ }^{15}$. La dictature s'assigne comme tâche de lutter contre la surpolitisation du pays responsable du chaos économique et social. Pour légaliser le coup d'Etat, 250 décretlois sont promulgués entre septembre 1973 et janvier $1974^{16}$, cette phase d'accumulation anarchique au gré des besoins ponctuels de la Junte perdure jusqu'à la

${ }_{12}$ DINGES, John, Les années Condor. Comment Pinochet et ses alliés ont propagé le terrorisme sur trois continents?, Paris, Ed. La Découverte, 2005.

13 Informe sobre la situación de los derechos humanos en Chile, Comisión Interamericana de Derechos Humanos, OEA/Ser.L/V/II.77.rev.1, Doc. 18, 8 mayo 1990, URL: < http://www.cidh.oas.org/countryrep/Chile85sp/indice.htm > [consulté le 14 de septembre 2015].

${ }^{14}$ Le général Pinochet chef d'Etat-major, le général Leigh chef de l'armée de l'air, l'amiral Merino chef de la marine et le général Mendoza chef des carabiniers.

${ }^{15}$ Message de la Junta de gobierno de las fuerzas armadas y carabineros de Chile radiodiffusé le 11 septembre 1973.

16 Informe de la Comisión Nacional de Verdad y Reconciliacion, vol. I, Santiago, La Corporación, 1991, pp. 55-70. 
fin de l'année 1975. Dorénavant l'application de la Constitution de 1925 est livrée à la volonté de la Junte qui s'attache à disposer des pleins pouvoirs pour mener à bien sa croisade contre le marxisme. Le cadre juridique imposé se caractérise par une rupture avec l'Etat de droit, le démantèlement des institutions préexistantes et la militarisation des rouages administratifs. Le Congrès est dissous, les registres électoraux sont déclarés caducs et les partis politiques interdits. Le pluralisme politique est révolu et tout retour à la démocratie par le biais d'élections est reporté sine die. Le nouveau régime exerce les pouvoirs exécutif et législatif; le respect de la constitution est circonscrit par le caractère des lois. En outre, tous les décrets-lois modifiant la loi fondamentale y sont incorporés conférant de fait un pouvoir constituant à la junte. L'administration est purgée; les services publics sont réorganisés tandis que les forces armées contrôlent les administrations locales et régionales. L'activité syndicale est supervisée par l'armée et les réunions à caractère politique sont interdites. Les recteurs d'universités en place sous l'Unité populaire sont limogés et remplacés par des recteurs délégués. L'Etat de siège est déclaré sur l'ensemble du territoire et l'Etat d'urgence imposé dans plusieurs provinces et départements, ces dispositions transfèrent les compétences de la justice ordinaire à des tribunaux militaires détenteurs de la pleine effectivité du pouvoir judiciaire; pour ce faire, elle bénéficie du laxisme de la Cour Suprême et des Cours d'Appels. La première décision précisant les relations entre les différents pouvoirs intervient en juin 1974 avec la promulgation du décret-loi $\mathrm{n}^{\circ} 527$, qui reconnaît une séparation formelle des pouvoirs: entre le président de la junte, chef de l'exécutif et la junte qui contrôle le législatif, tout cela dans le cadre de la Constitution de 1925. Il faut attendre le mois de janvier 1976 et l'élaboration des Actes constitutionnels pour que les fondements des nouvelles institutions soient définis ${ }^{17}$. En juillet 1977, lors du discours de Chacarillas, le général Pinochet pose les lignes du futur régime $^{18}$. Mais ces dernières doivent également considérer les effets du terrorisme d'Etat, c'est pourquoi le régime décide d'adopter une législation ad hoc sur la question de la violence politique. Le 10 mars 1978, est promulgué le décret-loi, ${ }^{\circ} 2191$, appelé «loi d'amnistie» dont le préambule est particulièrement éloquent:

la tranquillité générale, la paix et l'ordre dont jouit actuellement tout le pays font que les troubles intérieurs ont été surmontés et que, partant, il a été possible de mettre fin à l'état de siège et au couvre-feu sur tout le territoire national. L’impératif moral exige de mener à bonne fin les efforts visant à renforcer les

\footnotetext{
17 PINOCHET, Augusto, op. cit., p. 3.

18 CAVALO CASTRO, Ascanio, SALAZAR SALVO, Manuel, SEPULVEDA PACHECO, Oscar, La historia oculta del regimen militar, Santiago, Editorial Antartica, 1988, p. 161.
} 
liens qui unissent la Nation, en oubliant les haines aujourd'hui dépourvues de sens et en encourageant toutes les initiatives visant à consolider la réconciliation des Chiliens. La nécessité d'une unité nationale inébranlable s'est faite sentir pour permettre la mise en place progressive du nouveau système institutionnel qui doit régir le destin du Chiliip.

En ce sens, toute personne qui a participé à des actes délictueux pendant la durée de l'état de siège est amnistiée sous réserve qu'elle ne fasse pas l'objet d'une action judiciaire ou d'une condamnation; ce texte n'a toujours pas été abrogé. L'année suivante, le décret-loi $n^{\circ}$ 2621, rebaptisé «loi antiterroriste», institue une présomption de culpabilité et facilite la surveillance de la population et prévient tout réveil d'une opposition politique ${ }^{20}$.

Sur cette même période, la dictature se personnalise, une mutation qui préfigure le stade de la consolidation. Pour Verónica Valdivia Ortiz de Zárate, cette personnalisation du régime tient à deux facteurs:

l'ascendance prise sur les officiers Leigh et Merino qui ont préparé le coup d'Etat et qui font partie de la junte de gouvernement et la capacité de Pinochet à construire un leadership reposant sur un important soutien civil [et] elle est facilitée par le rapide isolement du nouveau gouvernement, lié aux graves violations des droits de l'homme, [...] elle est favorisée par les relations de Pinochet avec le noyau dur des putschistes. [...] Le phénomène est lié à la pensée politique de l'armée au moment du coup d'Etat qui permet d'éviter une démobilisation totale de la sociétée ${ }^{1}$.

Avec le décret-loi ${ }^{\circ} 527$ du 26 juin 1974, le général Pinochet s'empare du pouvoir exécutif en étant désigné chef suprême de la nation puis, le 17 décembre 1974, président de la République. Outre, sa volonté d'institutionnaliser son pouvoir, il va renouveler le haut commandement en nommant des hommes loyaux car son entrée tardive dans le complot constitue un élément de faiblesse face aux principaux protagonistes ${ }^{22}$. En ce

19 Concede amnistia a las personas que indica por los delitos que señala, Ministerio del Interior, 19 abril 1978, Biblioteca del Congreso Nacional de Chile, URL:

$<$ http://www.leychile.cl/Navegar?idNorma=6849 > [consulté le 23 septiembre 2015].

${ }^{20}$ Modifica D.F.L. (G) $\mathrm{n}^{\circ}$ 1, de 1968, y otras disposiciones legales, Ministerio del Interior, 28 abril 1979, Biblioteca del Congreso Nacional de Chile, URL:

< http://www.leychile.cl/Navegar?idNorma=6967 > [consulté le 23 septiembre 2015].

${ }^{21}$ VALDIVIA ORTIZ DE ZARATE, Verónica, «Construction du pouvoir et régime militaire sous Augusto Pinochet», op.cit., p. 94.

${ }^{22}$ GONZÁLEZ, Mónica, La conjura. Los mil y un días del golpe, Santiago, Ed. Catalonia, 2012, pp. 424-425. 
sens son succès passe par la mise au pas de l'institution militaire par le contrôle total du système des nominations et départs à la retraite des officiers. Par le décret-loi 33 du 21 septembre 1973, il peut durant une année nommer et écarter les officiers à a guise. Il en profite pour déplacer la plupart des officiers de sa génération susceptibles de défier son autorité comme le populaire général Bonilla, passé du ministère de l'Intérieur à celui de la défense en juillet 1974, qui mourra en 1975 dans un obscur accident d'avion. De surcroît il promeut l'extension du corps des généraux avec la nomination de jeunes officiers en fonction de leur loyauté envers leur chef et l'accès aux postes de pouvoir d'officiers issus de tous les corps d'armes²3. De sorte qu'à partir de 1977 l'ensemble des hauts gradés doivent leur promotion au général Pinochet. N'oublions pas que cette ascension au sein de la junte s'accomplit au moment où la DINA, qu'il dirige, est au faîte de sa puissance. L'année suivante, il doit affronter Gustavo Leigh sur le terrain politique lorsque ce dernier demande que soit défini un calendrier pour un retour à la démocratie représentative. La réponse est contenue dans le discours de Chacarillas et par la tenue d'un plébiscite de soutien au régime en 1978, contesté par le général Leigh qui est destitué de son poste au sein de la Junte. Le général Pinochet n'a plus d'opposants parmi le gouvernement, il détient tous les pouvoirs et l'armée est entièrement sous son contrôle. Le processus de centralisation est atteint avec l'étape de la constitutionnalisation.

\section{De la consolidation de la dictature pinochétiste à son issue légaliste (1979-1990)}

\subsection{Une nouvelle constitution institutionnalise le régime}

L’idée germe au moment du coup d'Etat. En effet, dès le 24 septembre 1973 est réunie à l'initiative du président de la junte de gouvernement, une commission pour étudier un nouveau projet de constitution de l'Etat. Et, le 11 octobre 1973, le général Pinochet affirme qu' «une nouvelle constitution politique de la République doit permettre une évolution dynamique conformément à l'évolution du monde moderne» ${ }^{24}$; le décret-loi $\mathrm{n}^{\circ}$ 1064, du 26 octobre 1973, officialise cette commission. Son

${ }_{23}$ BOISARD, Stéphane, Emergence d'une nouvelle droite au Chili: autoritarisme, monétarisme et conservatisme, 1955-1983, Thèse de doctorat, Université de Toulouse - Le Mirail, Toulouse, 2001, p. 393.

24 «Discurso del General Augusto Pinochet al conmemorar un mes del pronunciamiento militar», en Tercera de la Hora, Santiago, 12 octubre de 1973, cité par ORTEGA FREI, Eugenio, Historia de una alianza, Santiago, LOM, 1992, p. 151. 
travail devient effectif en 1977 lorsque le conseil d'Etat, instauré par le général Pinochet avec le premier acte constitutionnel de 1976 et présidé par l'ancien président Jorge Alessandri, recommande d'aller vers une constitutionnalisation du régime au-delà des seuls actes constitutionnels. Ladite commission envoie un projet au conseil d'Etat en octobre 1978 sur la base duquel ses membres élaborent une version du texte envoyée à A. Pinochet en juillet 1979. Ce dernier et ses assesseurs vont surtout modifier les normes transitoires dont la finalité est de le maintenir au pouvoir au minimum pendant huit ans ${ }^{25}$. Ainsi, le 11 août 1980 la junte approuve le projet définitif qui est accepté à la suite d'un simulacre de plébiscite démocratique. Face à une opposition désarticulée, uniquement représentée par le parti démocrate-chrétien et réorganisée autour du «Groupe des $24{ }^{26}$, l'absence de registres et de tribunaux électoraux et une propagande officielle incisive, le général Pinochet s'assure une victoire aisée en obtenant $67.06 \%$ de «oui».

Il est donc entendu que la constitution entrerait partiellement en vigueur en 1990 puis totalement en 1997 et il est envisagé que la junte puisse proposer un référendum en 1988. J. Samuel Valenzuela décline ce que l'on appelle la constitution de 1980 en deux temporalités consécutives à la constitution transitoire devant aboutir à l'élaboration d'une nouvelle loi fondamentale: la constitution permanente de la démocratie protégée et la constitution démocratique. La première cherche à refonder l'Etat de droit au Chili avec une mise en ordre constitutionnelle, présidentialiste et de fortes restrictions démocratiques. La seconde ne va finalement débuter qu'après le référendum de 1988.

Ce texte constitutionnel légitime, sous tous ses aspects, la dictature pinochétiste. Formellement, il est composé de 29 articles transitoires, qui prolongent et organisent le régime jusqu'en 1988, et de 120 articles permanents, l'ensemble visant à intégrer ce principe évolutif ${ }^{27}$. La répartition des pouvoirs assure la prééminence de l'exécutif avec une Chambre, comprenant 120 députés, désignés par la junte, et un Sénat, composé des anciens présidents de la République et de 41 personnes nommées par le chef de l'Etat. Le général Pinochet reste président de la République pour une période de 8 ans à partir de l'entrée en vigueur du texte, le 11 mars $1981^{28}$. De plus, il détient des pouvoirs

25 VALENZUELA, J. Samuel, «La Constitucíon de 1980 y el inicio de la Redemocratizacíon en Chile», in The Helen Kellogg Institue for International Studies, Working paper 242, Chicago, Université of Notre-Dame, 1997, p. 9.

${ }^{26}$ MOULIAN, Tomás, Chile actual, anatomía de un mito, Santiago, LOM, 2002, p. 236.

${ }_{27}$ Constitución política de la República de Chile, Honorable Cámara de Diputados de Chile, URL: < http://www.camara.cl/camara/media/docs/constitucion_politica.pdf > [consulté le 28 septembre 2015].

28 Ibidem, art.14 transitoire. 
considérables: restriction du droit de réunion et de la liberté d'information, interdiction de l'accès au territoire national aux individus prônant certaines doctrines, possibilité de mettre en détention toute personne en regard de son opinion politique, assignation à résidence ${ }^{29}$. Et, durant cette période, la junte de gouvernement continue d'exercer des fonctions constituantes et législatives ${ }^{30}$. Les articles permanents visent à assurer la survie du gouvernement militaire et la suprématie du général Pinochet. Pour ce faire, sont créées deux institutions spécifiques: le tribunal Constitutionnel pour établir une législation idoine et le conseil de sécurité nationale au sein duquel le général Pinochet et les membres de la junte ont la majorité. Dans la lignée de la phase répressive des années précédentes, la logique antisubversive est confirmée. La proscription idéologique est réaffirmée allant de l'inconstitutionnalité des formations de gauche ${ }^{31}$, à des dispositions relatives au terrorisme ${ }^{32}$, en passant par la déchéance de nationalité33, la possibilité de perdre le droit de vote 34 , l'opportunité de détenir une personne de cinq à dix jours avant de la mettre à la disposition d'un juge pour des actes qualifiés de terroriste 35 ou la séparation des activités politiques et syndicales ${ }^{36}$. Pour maintenir la paix sociale, quatre types d'états d'exception sont prévus ${ }^{37}$. En sus des garanties offertes par les articles transitoires, le président de la République se voit attribué des prérogatives immenses: droit de dissolution de la chambre des députés, désignation d'une partie des membres du Sénat, possibilité de décréter avec l'accord du conseil de sécurité nationale trois états d'exception, légiférer dans des domaines ne relevant pas expressément du domaine de la loi, nomination et mutation des gouverneurs régionaux et des maires des grandes villes, nomination des juges et de certains membres du tribunal constitutionnel et nomination et déplacement des commandants en chef des forces armées et du directeur général des carabiniers ${ }^{38}$. Le général Pinochet est dès lors l'«Homme fort du Chili» voire le «Maître du Chili»39. En outre, ce texte est la «pièce maitresse du programme politique du régime militaire» ${ }^{40}$ pour transformer la société

\footnotetext{
29 Ibidem, art.24 transitoire.

30 Ibidem, art.18 transitoire.

${ }^{31}$ Ibidem, art.8.

32 Ibidem, art.9.

33 Ibidem, art.11.

34 Ibidem, art.16.

35 Ibidem, art.19, paragraphe $7 \mathrm{c}$.

${ }^{36}$ Ibidem, art.23.

37 Ibidem, art.39.

$3^{8}$ Ibidem, art.32.

39 DE LA PARRA, Marco Antonio, Lettre ouverte à Pinochet: monologue de la classe moyenne chilienne avec son père, Paris, Le serpent à plumes, 1998, pp. 37-42.

40 VALENZUELA, J. Samuel, op. cit., p. 11.
} 
chilienne, influencé par celui de Jaime Guzmán et des Chicago Boys ${ }^{41}$. Les institutions vont fonctionner dans ce cadre constitutionnel jusqu'en 1988 sans déroger à leurs principes fondateurs en données corrigées des fluctuations conjoncturelles qu'elles soient économiques comme la crise financière et ses conséquences en 1982-1983, ou sociopolitiques à l'instar du mouvement des protestas de 1983-1984.

\subsection{Du référendum à la mise sous tutelle de la transition (1985-1990)}

A partir de 1985, sous l'égide de l'Eglise, l'opposition examine la possibilité de mettre fin au régime militaire par le biais du plébiscite prévu par la loi fondamentale, devant clôturer la première période présidentielle et insiste pour obtenir les garanties nécessaires à la tenue du scrutin ${ }^{42}$. Cette requête est validée par le tribunal constitutionnel mais doit être soumise au Tribunal calificador de elecciones, proche du pouvoir. Cette décision entérinée malgré la réprobation du gouvernement, le plébiscite peut être organisé avec une actualisation des registres électoraux et en permettant à l'opposition de s'exprimer quinze minutes par jour à la télévision pendant un mois. Le régime autorise cette mesure à une heure tardive afin de monopoliser le reste du temps d'écoute. Les semaines précédant la consultation sont marquées par une intense mobilisation pour le «Non»43. Notons que le général Pinochet accepte les règles constitutionnelles, probablement persuadé du soutien populaire et empreint d'une confiance excessive dans la victoire étant donné le contexte de croissance économique et des sondages officiels prédisant son succès. En conséquence, la nuit du 5 octobre 1988 est le moment de fortes tensions avec d'un côté, un gouvernement dictatorial prompt à clamer sa victoire, et de l'autre côté une opposition inquiète de l'attitude des militaires face à des résultats indiquant le contraire. Vers minuit, le général Pinochet convoque les commandants en chef des forces armées au siège du gouvernement afin de statuer sur le comportement à adopter, puis convient de façon laconique que la constitution doit s'appliquer44. Les forces de la Concertation remportent ce plébiscite avec 54,7\% des voix, résultat qui démontre également la popularité relative du général

\footnotetext{
${ }^{41}$ BOISARD, Stéphane, «Le général Pinochet et les Chicago Boys: les deux piliers de la dictature chilienne», in L'Ordinaire latino-américain, 193, 2003, pp. 43-54.

42 PROGNON, Nicolas, «L'Eglise chilienne face à la dictature: du compromis au rôle d'agent de la transition? (1973-1989)», in Cahier d'Histoire immédiate, 54, 2014, pp. 167-187.

43 Cette période d'effervescence sociale et politique est dépeinte dans le film LARRAIN, Pablo, No, Wild Side, Chili-France-Etats Unis, 2012, 117', inspiré de l'ouvrage de SKARMETA, Antonio, Los días del acoíris, Barcelona, Planeta, 2011.

44 CAVALO CASTRO, Ascanio, SALAZAR SALVO, Manuel, SEPULVEDA PACHECO, Oscar, op. cit., pp. 565-588.
} 
Pinochet. Le Chili entre dans une étape confuse de négociation entre les différents acteurs politiques au sujet de l'entrée en vigueur de la constitution démocratique. En effet, toute modification constitutionnelle requiert un accord entre le président et la junte ainsi que l'organisation d'un nouveau plébiscite.

De ce fait, le 30 juillet 1989, est confirmée, par 87,7 \% de votes favorables, la modification de 54 articles de la Constitution45. Cette réforme constitutionnelle octroie quelques concessions démocratiques ${ }^{46}$. Par ailleurs, les lois portant sur les États d'exception et les peines encourues pour une conduite terroriste sont rectifiées 47 . La dictature pinochétiste s'assure en contre-partie une forme de pérennité à travers cinq mécanismes : l'incorporation au Sénat de membres nommés par la Cour suprême, le président de la République et le conseil de sécurité nationale, et des ex-présidents de la République ; l'instauration d'un quorum élevé pour modifier la constitution ; la mise en place d'un système électoral minoritaire binominal; un quorum pour le vote des lois de «quorum qualifié» et «organiques constitutionnelles» des 4/7ème des députés et sénateurs en exercice ${ }^{48}$.

On retrouve l'esprit de ces mesures avec le verrouillage des systèmes politico institutionnels et juridiques avec le vote des «lois d'amarrage» en octobre 1989, qui enclavent la représentation parlementaire de l'opposition, les pouvoirs de l'exécutif et du Congrès dans les domaines jugés critiques pour les tenants du régime antérieur. Sept lois «organiques constitutionnelles» importantes, donc qui ne peuvent pas être modifiées sans un vote des 4/7ème des députés et sénateurs en exercice, vont règlementer plusieurs aspects politiques, institutionnels, administratifs et financier.

Tout d'abord, une loi établit comme mesure transitoire et exceptionnelle la suspension de la faculté de la Chambre des députés d'enquêter et de juger les actions des membres et fonctionnaires du gouvernement militaire, même dans les cas de corruption. De plus, le nouveau gouvernement doit maintenir en fonction les 30.000 fonctionnaires nommés sous la dictature et il ne lui est autorisé que la création de 400 nouveaux postes. Le futur président de la République se voit autorisé à nommer seulement 15 des 325 maires du pays; le reste est désigné par les Conseils de développement communal ou Codecos dont les membres seront placés par le général Pinochet avant son départ du pouvoir. La Banque centrale demeure autonome vis-à-vis

45 GUILLAUTAT, Patrick, MOUTERDE, Pierre, Les mouvements sociaux au Chili 1973-1993, Paris, L'Harmattan, 1995, pp. 218-220.

${ }^{46}$ Historia de la Ley $N^{o} 18.825$ Reforma de la Constitución Política de la República, Santiago, Biblioteca Nacional del Congreso de Chile, 1989, pp. 141-143.

47 Informe de la comisión nacional de verdad y de reconciliación, cit, pp. 76-77.

${ }^{48}$ GARRETON, Manuel Antonio, GARRETON, Roberto, «Las democracia incompleta en Chile: la realidad tras los rankings internacionales», in Revista de ciencia política, 30, 1/2010, p. 118. 
du pouvoir exécutif. Le système judiciaire est cadenassé avec une loi sur la régulation de la carrière des magistrats et du fonctionnement des tribunaux qui permet aux juges de la Cour suprême de prendre leur retraite avec une considérable indemnité de départ et au général Pinochet de choisir neuf nouveaux juges, soit une majorité des membres de la Cour. De son côté, l’institution militaire est choyée : une loi concernant les Forces Armées et la Police limite les facultés du futur président de la République en matière de nomination des commandants en chef des différentes armes, inamovibles sans le vote du Conseil de sécurité nationale. Le président de la République voit ses prérogatives limitées en matière de nomination, de promotion et de mise à la retraite des officiers et il perd ses facultés au niveau de la défense du territoire national. Cette autonomie préservée de l'armée est complétée par des garanties financières substantielles : le nouveau gouvernement doit assurer à l'armée un budget au moins égal, en termes réels, à celui de l'année 1989 et 10\% des ventes totales du cuivre réalisées par l'entreprise d'Etat Codelco. Les structures parallèles demeurent inchangées avec des services secrets qui restent sous le contrôle exclusif des commandants en chef de chaque branche. Enfin, les tribunaux militaires sont seuls aptes à juger des personnes liées à l'institution. Pour se prémunir de toute déroute politique de la droite qui pourrait signifier une réforme constitutionnelle en profondeur, la loi électorale établit un système unique pour les élections parlementaires et sénatoriales: le scrutin binominal par circonscription électorale et bi majoritaire par liste. Avec ce système, une liste peut donc espérer remporter la moitié des sièges lors d'une élection, tout en étant minoritaire. En « travestissant » l'ordre institutionnel, ce système électoral établit une stabilité polémique fondée autour de deux grands pôles électoraux car pour dégager une véritable majorité, un pôle doit avoir deux fois plus de voix que son adversaire ${ }^{49}$. Par ailleurs, le découpage électoral favorise les zones rurales plus conservatrices au détriment des grandes villes, en termes de nombre de parlementaires ${ }^{50}$.

En outre, ces dispositions se superposent à l'appareil juridique existant qui protège les auteurs de torture avec, notamment, la « loi d'amnistie » et les articles de la constitution de 1980 qui permettent au général Pinochet de rester commandant en chef des armées jusqu'en 1998, puis de devenir sénateur à vie. De ce fait, cette étape de la transition vers la démocratie est marquée par la toute-puissance de l'ancien dictateur qui dispose d'une impunité totale. Nonobstant le musellement constitutionnel du pays, le général Pinochet fait des concessions timides au sujet des droits de l'homme qui ne

49 CUADROS GARLAND, Daniela, «La démocratie chilienne (1990-2005) face à la cause des droits de l'homme et au désengagement des militaires», in Problèmes d'Amérique latine, 56/2005, pp. 40-42.

50 VALENZUELA, J. Samuel, op.cit., pp. 26-27. 
peuvent cependant pas déroger au legs autoritaire. Dans l'orbite de l'ancien président, les règles juridiques, mises en place entre 1978 et 1990, font de l'armée un acteur capable d'exercer un droit de regard sur le fonctionnement des institutions politiques et d'intervenir si la situation l'impose.

\section{Le démantèlement progressif, mais inachevé, du legs pinochétiste}

\subsection{Le lent déblocage du contexte politique}

Les différents gouvernements de transition présidés respectivement par Patricio Aylwin (1990-1994), Eduardo Frei Ruiz-Tagle (1994-2000), sont confrontés au blocage du système politique qui protège les auteurs militaires d'exactions commises entre 1973 et 1989 et pérennise la mainmise de l'ancien dictateur. La simple existence d'une telle menace suffit, pendant près d'une décennie, à éviter toute confrontation entre le gouvernement démocratique et le commandant en chef des forces armées. Elle permet, en outre, à ce dernier, de feindre l'ouverture en mystifiant la démocratie renaissante au sujet des droits de l'homme. Pour autant, le vecteur de cette transition sous tutelle de l'armée est le fruit d'un manque de courage politique des présidents chiliens car, comme le montrent les travaux de Claudio Fuentes sur les rapports de pouvoir entre les gouvernements de transition et l'armée, le président détient le droit de veto sur la promotion des officiers de l'armée, il peut restreindre le budget de l'armée, il peut se prononcer sur la viabilité d'achat de matériel militaire et il a le pouvoir de confirmer, ou non, les programmes de défense nationale ${ }^{51}$. Autant de dispositions que les présidents n'osent utiliser par crainte d'un conflit ouvert avec l'institution chaperonnée par l'ancien dictateur. Le mandat d'Eduardo Frei, de 1994 à 2000, tisse des relations de coopération avec les commandants en chef, avec une politique de modernisation de l'armée, sans se montrer trop regardant sur les rouages internes de l'institution. L'observation de la première décennie de la transition laisse entrevoir une seule faille dans le système légué par le général Pinochet: la vérité sur les violations des droits de l'homme perpétrées entre 1973 et 1990.

Ce dernier n'a pas pris en compte l'évolution de la justice transitionnelle ${ }^{2}$ et en dépit du pragmatisme de la transition, les familles des victimes ont déposé des plaintes

${ }^{51}$ FUENTES, Claudio, «After Pinochet: Civilian Policies Toward the Military in the 1990's Chilean Démocracy», in Journal of interamerican studies and World Affairs, 42, 3/200o, pp. 111-142.

${ }^{52}$ HAZAN Pierre, Juger la guerre, juger l'histoire, Paris, PUF, 2007, p. 174. 
contre lui entre 1992 et 1994, complétées en juillet 1996, par des recours enregistrés en Espagne. C'est pourquoi, en janvier 1998, le juge Juan Guzmán Tapia est désigné pour enquêter sur ces plaintes; la procédure trouve un prolongement inattendu avec l'arrestation du général Pinochet, le 16 octobre 1998, à Londres, alors qu'il venait subir une opération de hernie lombaire. Cette décision fait suite à une demande d'extradition notifiée par les juges espagnols Balthazar Garzón et Manuel Castellón García au motif de «génocide, torture, terrorisme et crimes contre l'humanité». Le 24 mars 1999, l’immunité du général Pinochet est levée. Malgré cette - apparente - avancée juridique, Jack Straw, ministre de l'intérieur de Grande Bretagne, s'oppose à l'extradition du général vers l'Espagne le jugeant inapte, le 2 mars 2000; le 3 mars, il peut rentrer au Chili où il est acclamé par les forces armées et ses alliés politiques. L'affaire, dite «Pinochet», donne une image désastreuse à l'étranger d'un pays désireux de développer ses relations économiques avec l'ensemble de la communauté internationale.

Ce changement d'échelle modifie profondément la manière dont la cause est élaborée : à partir du moment où les médias du monde entier s'emparent de l'affaire, les acteurs de la dénonciation sont confrontés à la nécessité de convaincre l'opinion internationale et non plus seulement l'opinion chilienne confrontée à sa propre histoire53.

Une révision des fondamentaux politiques nés des tractations de 1989 s'impose. La droite, aussi bien Rénovation nationale que l'Union démocratique indépendante, ainsi que la hiérarchie militaire doivent désormais adopter une attitude nouvelle face à ce passé embarrassant; l'immobilisme corporatiste vacille devant les inculpations répétées que doit affronter Augusto Pinochet. Pour sortir de l'impasse politique dans laquelle se trouve le gouvernement de transition dirigé par Ricardo Lagos, le 25 mars 2000, le Congrès adopte une réforme constitutionnelle visant à accorder l'immunité à Augusto Pinochet pour l'inciter à démissionner de son poste de sénateur. Les Chiliens doivent réapprendre la culture démocratique dans un pays qui a perdu confiance dans la politique et la transition est alors perçue comme «lente et douloureuse. Avec des lois en ruine, un simulacre de débats, avec beaucoup, beaucoup de mensonges, et des gesticulations de puissance lancées comme par des mains de noyés»54 et la démocratie comme «un marché [où] le journalisme est de la propagande et la publicité est la

53 COMPAGNON, Olivier, «L'affaire Pinochet : la démocratie chilienne dans le miroir de la justice », in Cahiers des Amériques, 46, 2004, pp. 49-61.

54 DE LA PARRA, Marco Antonio, op.cit., p.75. 
science occulte du pouvoir» 55 , ce régime placé dans la continuité du régime autoritaire, avec des règles institutionnelles léguées par le général Pinochet, hypothèque son avenir démocratique.

C'est pourquoi le président Lagos propose la création de la «Table pour le dialogue sur les droits de l'homme» afin d'aboutir à la réconciliation politique de la société chilienne ${ }^{56}$. Les protagonistes aboutissent à la signature par les militaires d'un document de consensus où ils reconnaissent publiquement la «violation des droits de l'homme» qu'ils avaient systématiquement niée auparavant et la désignation de juges spéciaux pour traiter des dossiers traitant des cas non résolus. Progressivement, ce rapprochement entre autorités civile et militaire induit l'isolement du cas Pinochet. Son arrestation pousse également les partis de droite à la rénovation de leur idéologie et de leur discours; selon Emmanuel Barozet, «l'un des aspects fondamentaux de l'évolution des droites chiliennes au cours de ces dernières années est leur capacité à prendre leurs distances par rapport à l'héritage du régime militaire»57. Ce processus met un certain temps à s'affirmer, notamment au sein de la UDI, formation tenante de la nouvelle droite chilienne soutien civil de l'ancien dictateur, jusqu'en 1999, lorsque Joaquin Lavín, futur candidat à l'élection présidentielle, rend une visite de courtoisie à Augusto Pinochet au nom de l'union sacrée des partis de droite. Dès lors, le soutien populaire à la UDI - et la droite en général - s'amoindrit. Une évidence s'impose : l'ancien dictateur ne fédère plus ses anciens réseaux clientélistes et la perspective d'une victoire électorale pour la droite passe inéluctablement par une rupture idéologique avec le régime militaire, sans pour autant revisiter les dogmes ultralibéraux.

\subsection{Un désengagement mesuré des forces armées marqueur de la fin de l'héritage dictatorial}

Au-delà du délitement de ses soutiens politiques, l'ancien caudillo est confronté à une nouvelle donne inhérente à une hiérarchie militaire moins encline à nier les excès de la période dictatoriale et ses contingences politiques. La nomination du général Cheyre à la tête de l'institution militaire en 2002 est un tournant; ce dernier décide de reconnaître la responsabilité de l'armée dans les violations des droits de l'homme. La position des militaires rompt partiellement avec le discours officiel lorsque, le 5

\footnotetext{
55 Ibidem, p. 81.

56 «Acuerdo de la Mesa de diálogo sobre derechos humanos», in Revista de Estudios Públicos, 79, 2000, pp. 481-487.

57 BAROZET, Emmanuel, «Les droites chiliennes: le dilemme de la rénovation», in Problèmes d'Amérique latine, 56, 2005, pp. 15-35, p. 23.
} 
novembre 2004, dans un document intitulé «Armée chilienne: la fin d'une vision», le général Juan Emilio Cheyre annonce: «l'armée chilienne a pris la dure mais irréversible décision et moralement inacceptable d'un passé [génant]»58. En assumant symboliquement leur culpabilité, les responsables de l'armée n'en admettent pas moins que les crimes sont le fait de comportements individuels déviants; nonobstant ce conformisme, on avance lentement vers la reconnaissance d'une politique systématique de violations des droits de l'homme. Ce revirement du commandement en chef provient de la publication par le président Lagos du rapport de la Commission nationale sur la prison politique et la torture, instituée en 2003 dans la lignée des décisions prises lors de la Table de dialogue 59. De fait, l'institution décide de rentrer dans les casernes et de délaisser le terrain politique que les lois d'amarrage leur avait permis d'occuper jusqu'alors et pour peser sur le processus transitionnel.

Dans ce contexte, le président Ricardo Lagos propose une réforme constitutionnelle visant à réduire le fardeau inhérent au legs autoritaire. Ces modifications mettent en exergue la fin de la tutelle des forces armées et par voie de conséquence, sonnent le glas du personnage politique Pinochet. Dans la nuit du 13 juillet 2005, le Sénat vote à la majorité des amendements à la constitution de 1980 réduisant de six à quatre ans le mandat du président de la République et éliminant, de facto, les statuts de sénateur désigné et de sénateur à vie, en même temps qu'ils restituent au pouvoir civil la faculté de changer les commandants des forces armées et de la gendarmerie. Le pouvoir civil dispose désormais du pouvoir décisionnel nécessaire pour intervenir dans des domaines auparavant dévolus au Conseil de sécurité nationale dont les prérogatives se limitent à donner des conseils et des recommandations. Ces dispositions entérinées en séance plénière, le 16 août 2005, par le Congrès de Valparaiso, entrent en vigueur le 11 mars 2006, jour de l'intronisation de Michelle Bachelet, nouvelle présidente de la République. Malgré ces avancées, certains points du legs pinochétiste sont des charges pour le pouvoir. La mort du général Pinochet, le 10 décembre 2006, referme définitivement la parenthèse des années de plomb comme le laissent augurer certains éléments. D’une part, elle n'a eu aucune résonance sur le déroulement de la vie politique nationale car la Présidente Bachelet n'a pas modifié son agenda et elle a refusé que l'ancien Chef de l'Etat bénéficie de funérailles nationales tout en réaffirmant le besoin de ne pas oublier pour que la société chilienne puisse recouvrer une mémoire collective et se réconcilier. D’autre part, les seuls honneurs publics reçus par Augusto

58 «L'armée chilienne reconnaît ses crimes pendant la dictature», Le Monde, 5 novembre 2004. 59 COMISION NACIONAL DE PRISON POLITICA Y TORTURA, Informe Comisión Nacional sobre Prisión Política y Tortura, Santiago, s.e., 2004, p. 15. 
Pinochet ont été un hommage de l'armée et une retransmission en direct à la télévision. Le temps des grandes parades militaires et de la déférence empruntée des gouvernements de transition est révolu.

Coïncidence ou non, pour la première fois la Cour suprême statue sans tenir compte de la loi d'amnistie en appuyant sa décision sur une disposition de la Cour interaméricaine qui a condamné le décret-loi nº 2191 de 1978. La justice n'est plus aux mains de l'armée et dès 2007 , le Chili va se doter d'outils du droit international qui permettent de contourner la loi d'amnistie. En 2008, le gouvernement ratifie le Protocole Facultatif de la Convention contre la torture et autres traitements cruels, inhumains et dégradants del'ONU et, en 2009, il intègre dans sa législation la notion de crimes de lèse humanité et il promulgue les statuts de Rome de la Cour Pénale Internationale et ceux du Protocole contre la Torture. Le caractère international de la justice transitionnelle s'est imposé formellement à l'amnistie et au fonctionnement déficient en la matière de la justice chilienne. L'avenir de celle-ci, pour refermer l'héritage pinochétiste, passe maintenant par son internationalisation. Tant que la Chambre des députés n'aura pas abrogé la loi d'amnistie qui, avec la loi antiterroriste appliquée à l'encontre des Mapuches ${ }^{60}$, doit tomber pour rendre à la justice la dignité tant de fois demandée par les victimes, en condamnant les responsables et faire du Chili un Etat de droit exemplaire en Amérique latine. De plus d'autres vestiges autoritaires pèsent sur le pays comme les systèmes éducatif et économique des plus inégalitaires au monde. Mais l'actuel mandat de Michelle Bachelet laisse augurer la mise à bas de ce legs constitutionnel en proposant la gratuité de l'enseignement, la réforme fiscale et une nouvelle constitution. De la sorte, le vote par le sénat, le 15 janvier 2015, de la fin du système binominal, qui devrait être ratifié par un vote de la Chambre des députés où la Concertation est majoritaire, pourrait devenir un tremplin vers la mise en place d'une assemblée constituante qui sonnerait le glas de la loi fondamentale en vigueur.

Depuis le 11 septembre 1973, la dictature pinochétiste a fait preuve d'une longévité inaccoutumée en occupant le pouvoir jusqu'en décembre 1989. De plus, elle a montré sa capacité à peser sur la gouvernance du pays tout en n'occupant plus de fonctions politiques directes depuis. La violence de l'intervention des forces armées cumulée à la volonté sans borne du général Pinochet de marquer l'histoire nationale, et sa propension, en accord avec les forces partisanes alliées, à institutionnaliser son pouvoir, en font une dictature atypique en comparaison de ses homologues du Cône

6o BENGOA, José, «Los Mapuches. Historia, cultura y conflicto», in Cahiers des Amériques latines, 68, 2/2012, pp. 89-107. 
sud. En institutionnalisant le régime par étape, elle a acquis une légitimité - certes critiquable - et a été le socle de cette prégnance sur la société chilienne. Si notre propos n'était pas de développer son bilan en termes de violations des droits humains et sur la révolution néolibérale engagée de façon autoritaire, il est manifeste que ces deux facteurs ont contribué à la réussite du projet initié au lendemain du coup d'Etat. En ce sens, l'alliance entre civils et militaires a garanti, en partie, à la dictature son impunité au-delà du plébiscite du 5 octobre 1988. Plus de quarante ans après, le Chili subit encore les convulsions de cet héritage non assumé par l'ensemble de la société. La réconciliation négociée avec le général Pinochet et pragmatique acceptée par les gouvernements depuis 1990 paraît aujourd'hui impossible tant elle est la contingence d'une quête irrépressible de démocratie visant à remplacer un régime autoritaire, qui la maintient sous tutelle, et à «y substituer des procédures de légitimation exigeant un temps long, scandé par les échéances électorales et privilégiant la transformation par construction ${ }^{61}$. Néanmoins les dernières élections présidentielles ont montré, à travers l'alternance de droite en 2010 avec le mandat de Sebastián Piñera Echenique et la réélection de Michelle Bachelet en 2014, la solidité de cette démocratie qui peut avancer sans la pression des forces armées. Cependant, l'héritage pinochétiste, bien que graduellement défait, est un point de discorde au Chili.

${ }^{61}$ LEFRANC, Sandrine, Politiques du pardon, Paris, PUF, 2002. 


\section{${ }^{*}$ L'auteur}

Nicolas Prognon est docteur en histoire de l'Amérique latine, enseignant, chercheur et membre du GRHI de l'Université Jean Jaurès de Toulouse et du groupe Histoire en Débat de SaintJacques-de-Compostelle (Espagne). Est auteur de plusieurs articles sur l'exil chilien, le retour, ses représentations en France, et sur l'histoire immédiate du Chili.

URL: < http://www.studistorici.com/progett/autori/\#Prognon >

\section{Per citare questo articolo:}

PROGNON, Nicolas, «Physionomies et héritages de la dictature pinochétiste : du terrorisme d'Etat à la mise sous tutelle du processus transitionnel (1973-2015)», Diacronie. Studi di Storia Contemporanea : Le dittature militari: fisionomia ed eredità politica, 29/12/2015,

URL:< http://www.studistorici.com/2015/12/29/prognon_numero_24/ >

Diacronie Studi di Storia Contemporanea $\vartheta$ www.diacronie.it

Risorsa digitale indipendente a carattere storiografico. Uscita trimestrale.

redazione.diacronie@hotmail.it

Comitato di redazione: Jacopo Bassi - Luca Bufarale - Elisa Grandi - Antonio César Moreno Cantano - Deborah Paci - Fausto Pietrancosta - Alessandro Salvador - Matteo Tomasoni - Luca Zuccolo

Diritti: gli articoli di Diacronie. Studi di Storia Contemporanea sono pubblicati sotto licenza Creative Commons 3.0. Possono essere riprodotti e modificati a patto di indicare eventuali modifiche dei contenuti, di riconoscere la paternità dell'opera e di condividerla allo stesso modo. La citazione di estratti è comunque sempre autorizzata, nei limiti previsti dalla legge. 